\title{
ASTEROIDAL QUADRUPLES IN NON ROOTED PATH GRAPHS
}

\author{
MARisa Gutierrez \\ Conicet \\ Dto. de Matemática Facultad de Ciencias \\ Exactas Universidad Nacional de La Plata \\ Argentina \\ e-mail: marisa@mate.unlp.edu.ar \\ BenJamin LÉvêQUE \\ CNRS, LIRMM, Montpellier, France \\ e-mail: benjamin.leveque@lirmm.fr \\ AND \\ Silvia B. Tondato \\ Dto. de Matemática Facultad de Ciencias \\ Exactas Universidad Nacional de La Plata \\ Argentina \\ e-mail: tondato@mate.unlp.edu.ar
}

\begin{abstract}
A directed path graph is the intersection graph of a family of directed subpaths of a directed tree. A rooted path graph is the intersection graph of a family of directed subpaths of a rooted tree. Rooted path graphs are directed path graphs. Several characterizations are known for directed path graphs: one by forbidden induced subgraphs and one by forbidden asteroids. It is an open problem to find such characterizations for rooted path graphs. For this purpose, we are studying in this paper directed path graphs that are non rooted path graphs. We prove that such graphs always contain an asteroidal quadruple.
\end{abstract}

Keywords: clique trees, rooted path graphs, asteroidal quadruples.

2010 Mathematics Subject Classification: 05C75. 


\section{REFERENCES}

[1] T. Kloks, D. Kratsch, and H. Muller, Asteroidal sets in graphs, Lecture Notes in Comput. Sci. 1335 (1997) 229-241.

doi:10.1007/BFb0024501

[2] K. Cameron, C.T. Hoáng and B. Lévêque, Asteroids in rooted and directed path graphs, Electron. Notes Discrete Math. 32 (2009) 67-74. doi:10.1016/j.endm.2009.02.010

[3] K. Cameron, C.T. Hoáng and B. Lévêque, Characterizing directed path graphs by forbidden asteroids, J. Graph Theory 68 (2011) 103-112. doi:10.1002/jgt.20543

[4] S. Chaplick, M. Gutierrez, B. Lévêque and S.B.Tondato, From path graphs to directed path graphs, Lecture Notes in Comput. Sci. 6410 (2010) 256-265. doi:10.1007/978-3-642-16926-7_24

[5] F. Gavril, The intersection graphs of subtrees in trees are exactly the chordal graphs, J. Combin. Theory Ser. B 16 (1974) 47-56. doi:10.1016/0095-8956(74)90094-X

[6] C.G. Lekkerkerker and J.Ch. Boland, Representation of finite graph by a set of intervals on the real line, Fund. Math. (1962) 45-64.

[7] B. Lévêque, F. Maffray and M. Preissmann, Characterizing path graphs by forbidden induced subgraphs, J. Graph Theory 62 (2009) 369-384.

doi:10.1002/jgt.20407

[8] I. Lin, T. McKee and D.B. West, The leafage of a chordal graphs, Discuss. Math. Graph Theory 18 (1998) 23-48. doi:10.7151/dmgt.1061

[9] C. Monma and V. Wei, Intersection graphs of paths in a tree, J. Combin. Theory Ser. B 41 (1986) 141-181. doi:10.1016/0095-8956(86)90042-0

[10] B.S. Panda, The forbidden subgraph characterization of directed vertex graphs, Discrete Math. 196 (1999) 239-256. doi:10.1016/S0012-365X(98)00127-7

Received 15 May 2014

Revised 16 December 2014

Accepted 16 December 2014 